\title{
Efficient Sunlight-Induced Methylene Blue Removal over One-Dimensional Mesoporous Monoclinic $\mathrm{BiVO}_{4}$ Nanorods
}

\author{
Linrui Hou, Long Yang, Jiaoyang Li, Jie Tan, and Changzhou Yuan \\ Anhui Key Laboratory of Metal Materials and Processing, School of Materials Science and Engineering, Anhui University of Technology, \\ Ma'anshan 243002, China \\ Correspondence should be addressed to Linrui Hou, linruihou@yahoo.cn and Changzhou Yuan, ayuancz@163.com
}

Received 25 November 2011; Revised 16 January 2012; Accepted 8 February 2012

Academic Editor: Kea-Tiong Tang

Copyright (๑) 2012 Linrui Hou et al. This is an open access article distributed under the Creative Commons Attribution License, which permits unrestricted use, distribution, and reproduction in any medium, provided the original work is properly cited.

Sunlight-driven mesoporous $\mathrm{BiVO}_{4}$ nanorods with monoclinic structure have been successfully synthesized via a simple hydrothermal method. The as-prepared one-dimensional $\mathrm{BiVO}_{4}$ nanorods exhibited high specific surface area due to their unique mesoporous structure. The mesoporous $\mathrm{BiVO}_{4}$ nanorods possessed strong photoabsorption properties in the visible light region as well as the ultravisible region, and the band gap was estimated to be $c a .2 .18 \mathrm{eV}$. The photocatalytic activities were evaluated by decolorization of methylene blue under sunlight irradiation. Photocatalytic tests demonstrated that the decolorization rate of as-prepared mesoporous $\mathrm{BiVO}_{4}$ nanorods was even up to $98.8 \%$ in $180 \mathrm{~min}$, much better than that prepared by solid-state reaction (23.1\%) and the commercial $\mathrm{TiO}_{2}$ (Degussa P25) (14.2\%) under the same conditions, due to their higher specific surface area and appropriate band gap. Moreover, the unique $\mathrm{BiVO}_{4}$ nanorods exhibit high stability after five photocatalytic degradation recycles.

\section{Introduction}

Organic dyes in textile and industrial effluents have become the major environmental contaminants. Furthermore, many dyes are highly water-soluble, and some traditional treatment methods, such as activated carbon adsorption, flocculation, and biological treatment, do not work efficiently [1]. Thus it has become a challenging and indispensable topic of modern research how to efficiently solve this urgent environmental issue. Recently, photocatalysis technique has displayed its obvious advantage in comparison to the above treatment methods. The early study mainly focused on the $\mathrm{TiO}_{2}$-based photocatalysts, while $\mathrm{TiO}_{2}$ only responds to ultravisible (UV) irradiation, which occupies only $c a .4 \%$ of the whole solar energy [2], which greatly hinders its further wider application. To utilize efficiently cheap solar energy, the development of sunlight-driven photocatalyst has become one of the most significant topics recently.

In the search of semiconductor materials with photocatalytic activity under sunlight irradiation, many efforts have been put on designing nontitania-based semiconductor photocatalysts including $\mathrm{CdBi}_{2} \mathrm{O}_{4}$ [3], $\mathrm{Bi}_{3} \mathrm{O}_{4} \mathrm{Cl}$ [4], and $\mathrm{Bi}_{2} \mathrm{MoO}_{6}$ [5], $\mathrm{Ag}_{2} \mathrm{CrO}_{4}[6], \mathrm{BiVO}_{4}[7,8]$, and $\mathrm{Bi}_{2} \mathrm{MoO}_{6}$
[9]. Those new ternary metal oxide semiconductors possess steep absorption edges in the visible-light region, which is obviously different from the structured spectrum of $\mathrm{TiO}_{2}$ [10]. In addition, compared to $\mathrm{TiO}_{2}$, the valence bands of the ternary metal oxide semiconductors consist of hybridizations of transition-metal orbitals, and this hybridization can increase the VB level, thus resulting in the narrow band gap. The narrower band gap of these photocatalysts will facilitate excitation of the electrons from the valence band (VB) to the conduction band (CB), which is beneficial to the photocatalytic oxidation reaction [11]. Moreover, for the photocatalytic degradation of dilute pollutants, the exposed surfaces of the photocatalysts will serve as centers of condensing substrates through a physical adsorption process, and the condensed substrates would be degraded by generated hydroxyl radical [12]. Therefore, a photocatalyst with large surface specific area (SSA) is also important for the efficient degradation of dilute pollutants.

As one of the nontitania-based visible-light-driven semiconductor photocatalysts, $\mathrm{BiVO}_{4}$ has attracted extensive interests for organic photocatalytic degradation due to its narrow band gap. $\mathrm{BiVO}_{4}$ crystallizes in three different 


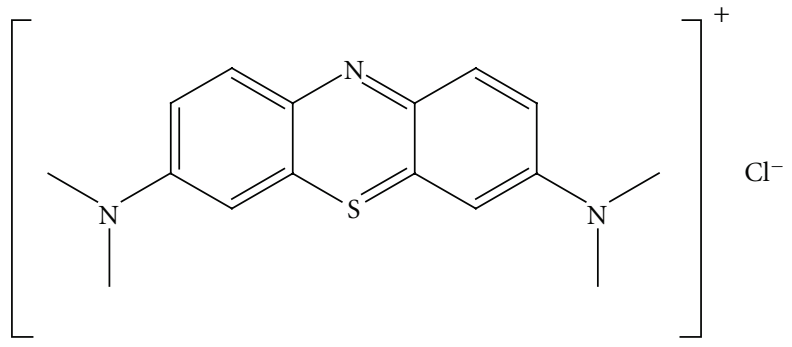

FIGURE 1: Structure of the methylene blue.

polymorphs, that is, tetragonal zircon, monoclinic distorted scheelite, and tetragonal scheelite, in which monoclinic $\mathrm{BiVO}_{4}$ shows the highest photocatalytic activity $[13,14]$. Thus, the synthesis of $\mathrm{BiVO}_{4}$ with monoclinic phase has become the focus of study today. Up to now, various methods have been used to synthesize $\mathrm{BiVO}_{4}$ with monoclinic phase, such as solid-state reaction method [15, 16], ultrasonicassisted microemulsion method [17], aqueous process [18, 19], flame spray pyrolysis [20], hydrothermal method [21], and solvothermal route [22]. Among these synthetic methods, hydrothermal method is found to be facile and efficient for the preparation of $\mathrm{BiVO}_{4}$ with monoclinic phase [21].

In addition, the discharge of colored wastewater from industries has caused many environmental problems, and many dyestuffs cannot be degraded by a conventional method, such as biological treatment. Among all the nonbiodegradable stuffs, methylene blue (MB) is a commonly used dyestuff that has wider applications including coloring paper, temporary hair colorant, dyeing cottons, wools, and coating for paper stock. However, acute exposure to $\mathrm{MB}$ will cause health problems, such as increasing heart rate, vomiting, shock, Heinz body formation, cyanosis, and tissue necrosis in humans [23-25]. For this reason, it is imperative to develop the photocatalysts for the effective degradation of $\mathrm{MB}$.

Based on the above overviews, in this paper, one-dimensional (1D) monoclinic $\mathrm{BiVO}_{4}$ nanorods with rich mesopores, to the best of our knowledge, were first synthesized by a facile hydrothermal method. The high sunlight-induced photocatalytic activity of $\mathrm{BiVO}_{4}$ nanorods was investigated based on $\mathrm{MB}$ as decomposed substance. For reference, the structure of $\mathrm{MB}$ is presented in Figure 1. Photocatalytic experiments demonstrated that $\mathrm{MB}$ was almost completely decomposed within only $180 \mathrm{~min}$ under sunlight irradiation, revealing their excellent photocatalytic activity under sunlight, which is of great significance to its practical applications.

\section{Experimental}

2.1. Preparation of Mesoporous $B^{2} V_{4}$ Nanorods. All the reagents were of analytical purity and used without further purification. The typical synthesis of mesoporous $\mathrm{BiVO}_{4}$ nanorods was shown as follows: $\mathrm{Bi}\left(\mathrm{NO}_{3}\right)_{3} \cdot 5 \mathrm{H}_{2} \mathrm{O}(3 \mathrm{mmol})$ and $\mathrm{NH}_{4} \mathrm{VO}_{3}(3 \mathrm{mmol})$ were firstly dissolved into $30 \mathrm{~mL}$ of dilute $\mathrm{HNO}_{3}$ aqueous solution $(2 \mathrm{M})$, respectively, and then two nitric acid solutions of $\mathrm{Bi}\left(\mathrm{NO}_{3}\right)_{3} \cdot 5 \mathrm{H}_{2} \mathrm{O}$ and $\mathrm{NH}_{4} \mathrm{VO}_{3}$ were mixed. Afterwards, the $\mathrm{pH}$ value of the mixed solution was adjusted to 6-7 by ethylenediamine. Finally, the precursor solution was transferred into a $100 \mathrm{~mL}$ Teflonlined stainless-steel autoclave and then heated at $160^{\circ} \mathrm{C}$ for $5 \mathrm{~h}$. Subsequently, it was cooled to room temperature, and the yellow precipitates were separated by filtration, washed with deionized water and absolute ethanol several times, and then dried at $60^{\circ} \mathrm{C}$ for $12 \mathrm{~h}$ in air. For comparison, another $\mathrm{BiVO}_{4}$ sample (denoted as SSR-BiVO ${ }_{4}$ ) was also prepared by a solid-state reaction according to the reference [26].

2.2. Characterization. The crystalline phase and image of the sample were determined by X-ray diffractometer (XRD, Max $18^{\mathrm{XCE}}$, Japan) using a Cu Ka source $(\lambda=0.154056 \mathrm{~nm})$, scanning electron microscopy (SEM, LEO 1403VP, Germany), and transmission electronic microscopy (TEM, Hitachi-600, Japan), respectively. To determine the band gap energy of the photocatalyst, UV-Vis diffuse reflectance spectrum was carried out, in the wavelength range of $200-800 \mathrm{~nm}$, using a Hitachi U-3010 spectrophotometer. The pure powdered $\mathrm{Al}_{2} \mathrm{O}_{3}$ was used as a reference sample. $\mathrm{N}_{2}$ adsorption/desorption was determined by Brunauer-Emmett-Teller (BET) measurements using an ASAP-2010 surface area analyzer.

\subsection{Adsorption Behavior of $M B$ on the Synthesized Photo-} catalysts. To determine the adsorption behavior of $\mathrm{MB}$ on the synthesized photocatalysts, the typical preparation of the suspensions was as follows. Three given weights $(0.200 \mathrm{~g})$ of different photocatalysts were mixed in three aliquots of $\mathrm{MB}$ solutions $(100 \mathrm{~mL})$ with the same initial concentrations $\left(5 \times 10^{-5} \mathrm{~mol} / \mathrm{L}\right)$, respectively. The suspensions were kept overweight in the dark and filtered after being centrifuged. The absorbance of the filtrated was then measured at the maximum band of $464 \mathrm{~nm}$ to determine the concentration of $\mathrm{MB}$. The extent of equilibrium adsorption was determined from the decrease in the MB concentration detected after filtration. The Brunauer-Emmett-Teller (BET) surface area was estimated by using $\mathrm{N}_{2}$ adsorption data on a Micromeritics Tristar 3020 apparatus.

2.4. Evaluation of Photocatalytic Activity. All photocatalytic experiments were carried out under similar conditions in summer months of July and August. The sky is clear, and the sunrays are very intense in this period in the city of Urumqi (latitude: $43.46 \mathrm{~N}$; longitude: 87.36 E), Xinjiang (China). All the solar experiments were performed on bright sunny days in order to avail maximum sunshine.

The photocatalytic activity of mesoporous $\mathrm{BiVO}_{4}$ nanorods was evaluated by photocatalytic degradation of $\mathrm{MB}$ aqueous solution. All photocatalytic reactions were performed under sunlight irradiation in a quartz beaker. The as-prepared photocatalyst $(0.200 \mathrm{~g})$ was dispersed in $100 \mathrm{~mL}$ of $\mathrm{MB}$ solution with initial concentration of $5 \times 10^{-5} \mathrm{~mol} / \mathrm{L}$ by stirring with a magnetic stirrer. The solution was stirred for $30 \mathrm{~min}$ in dark to allow the system to reach an adsorption/desorption equilibrium, then after a predetermined time, $5 \mathrm{~mL}$ of the solution was drawn and centrifugated. 


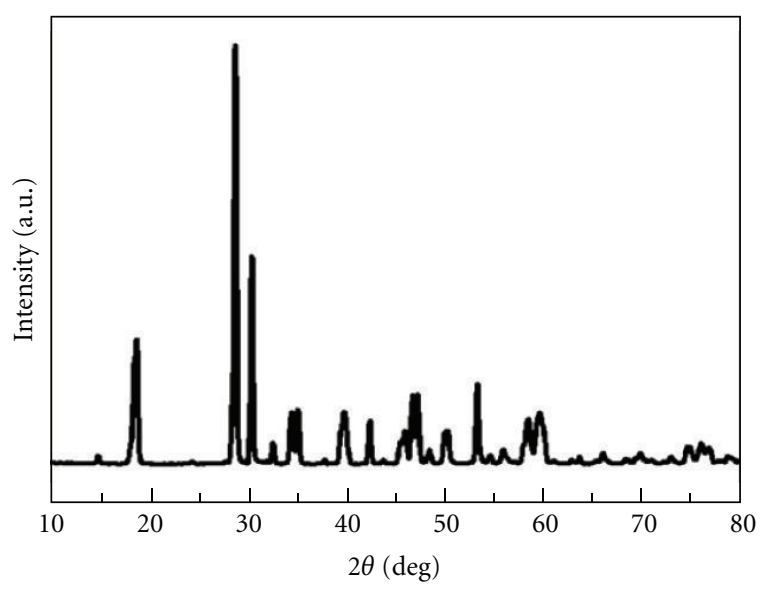

FIGURE 2: XRD pattern of the as-prepared mesoporous $\mathrm{BiVO}_{4}$ nanorods.

The optical absorption spectrum for the supernatant solution was recorded using a double-beam spectrophotometer (UV-2450, Japan and absorption at $\lambda_{\max }=664 \mathrm{~nm}$ for $\mathrm{MB})$. The decolorization rate of $\mathrm{MB}$ was calculated by the following equation $[27,28]$ :

$$
D=\frac{C_{0}-C}{C_{0}} \times 100 \%=\frac{A_{0}-A}{A_{0}} \times 100 \%,
$$

where $C_{0}$ and $A_{0}$ were the concentration and absorbency of $\mathrm{MB}$ solution at maximum absorption wavelength of the initial solution and $C$ and $A$ were the concentration and absorbency of $\mathrm{MB}$ solution at maximum absorption wavelength after sunlight irradiation at any time.

For comparison, a $250 \mathrm{~W}$ medium pressure mercury arc lamp with a main wavelength of $450 \mathrm{~nm}$, was also used in the experiments to replace natural sunlight to investigate the photocatalytic properties of all the products in the laboratory in a similar quartz beaker.

\section{Results and Discussion}

3.1. Physicochemical Characterization of the As-Prepared $\mathrm{BiVO}_{4}$ Sample. The phase and composition of the asprepared product were investigated by using XRD measurement. Figure 2 shows the XRD pattern of $\mathrm{BiVO}_{4}$ powder sample prepared by hydrothermal method at $160^{\circ} \mathrm{C}$ for $5 \mathrm{~h}$. All diffraction peaks can be indexed to be a monoclinic phase $\mathrm{BiVO}_{4}$ (JCPDS no. 83-1699), and no peaks for any other phases or impurities are detected.

The morphology and microstructure of the as-prepared $\mathrm{BiVO}_{4}$ were revealed by SEM and TEM, as shown in Figure 3. The SEM images (Figures 3(a) and 3(b)) clearly demonstrate that the as-prepared products are almost entirely rods with length of $0.5-2 \mu \mathrm{m}$ and diameter of $50-80 \mathrm{~nm}$. The rodlike morphology of the products is further confirmed by TEM images (Figure 3(c)), and the close-up observation of the samples revealed by the high-magnification TEM image (Figure 3(d)) shows that the rod possesses a porous interior
TABLE 1: BET SSA and reaction rate constant $k$ of mesoporous $\mathrm{BiVO}_{4}$, nanorods, $\mathrm{SSR}_{-} \mathrm{BiVO}_{4}$ and $\mathrm{P} 25$, respectively.

\begin{tabular}{lcc}
\hline Sample & $\mathrm{SSA}_{\mathrm{BET}}\left(\mathrm{m}^{2} / \mathrm{g}\right)$ & $k\left(\mathrm{~min}^{-1}\right)$ \\
\hline $\mathrm{BiVO}_{4}$ nanorods & 10.67 & 0.0243 \\
$\mathrm{SSR} \mathrm{BiVO}_{4}$ & 0.748 & 0.0015 \\
$\mathrm{P} 25$ & 50 & 0.0009 \\
\hline
\end{tabular}

structure, which increases the SSA of these nanorods and their photocatalytic reaction. Up to now, $\mathrm{BiVO}_{4}$ nanorods have been prepared by many groups [29-31], while $\mathrm{BiVO}_{4}$ nanorods with pores were seldom synthesized. During the reaction process, ethylenediamine plays an important role, and it not only adjusts the $\mathrm{pH}$ value of the mixed solution, but also directs the assembly of nanoparticles. A possible formation mechanism for mesoporous $\mathrm{BiVO}_{4}$ nanorods was tentatively illustrated. At first, the precursor formed by the reaction between $\mathrm{BiO}^{+}$and $\mathrm{VO}_{3}{ }^{-}$ions when the $\mathrm{pH}$ value of the mixed nitric acid solutions of $\mathrm{Bi}\left(\mathrm{NO}_{3}\right)_{3} \cdot 5 \mathrm{H}_{2} \mathrm{O}$ and $\mathrm{NH}_{4} \mathrm{VO}_{3}$ was adjusted to 6-7 by ethylenediamine. When the precursor solution was treated under the hydrothermal condition at $160^{\circ} \mathrm{C}$, the nanoparticles rearrange orderly to obtain rod-like structure under the direct of ethylenediamine. As for the formation of pores, it is probably attributed to the noncompactly accumulated nanoparticles.

The SSA of all photocatalysts in this research also has been listed in Table 1. As shown in Table 1, SSR-BiVO displays a much lower SSA of $0.748 \mathrm{~m}^{2} / \mathrm{g}$, while the as-prepared mesoporous $\mathrm{BiVO}_{4}$ nanorods exhibit a significantly higher SSA of $10.67 \mathrm{~m}^{2} / \mathrm{g}, c a$. 14 times higher than SSR$\mathrm{BiVO}_{4}$. For commercial $\mathrm{TiO}_{2}$ (Degussa P25), its SSA is $50 \mathrm{~m}^{2} / \mathrm{g}$ [28]. The larger exposed surfaces of the mesoporous $\mathrm{BiVO}_{4}$ nanorods will serve as centers of condensing $\mathrm{MB}$ through a physical adsorption process, and the condensed MB would be degraded by generated hydroxyl radical [12].

In general, the optical absorption property and migration of the light-induced electrons and holes of a semiconductor are recognized as the key factors in determining its photocatalytic activity [32]; thus it is the ideal way guiding the synthesis of semiconductors with good optical absorption property. Figure 4 shows the room temperature UV-Vis diffuse reflectance spectrum of mesoporous $\mathrm{BiVO}_{4}$ nanorods with steep shape, indicating that the visible light absorption is ascribed to the band gap transition [33]. The mesoporous $\mathrm{BiVO}_{4}$ nanorods show strong photo-absorption property not only in the UV region but also in the visible light region until $600 \mathrm{~nm}$. With respect to the wavelengths of absorption edges, they are determined by extrapolating the horizontal and sharply rising portions of the curves and defining the edge as the wavelength of the intersection [34]. The energy of the band gap of $\mathrm{BiVO}_{4}$ can be calculated as ca. $2.18 \mathrm{eV}$ from its band gap absorption edge, which means that the as-prepared $\mathrm{BiVO}_{4}$ sample has a suitable band gap for photocatalytic decomposition of organic contaminants under sunlight irradiation. 


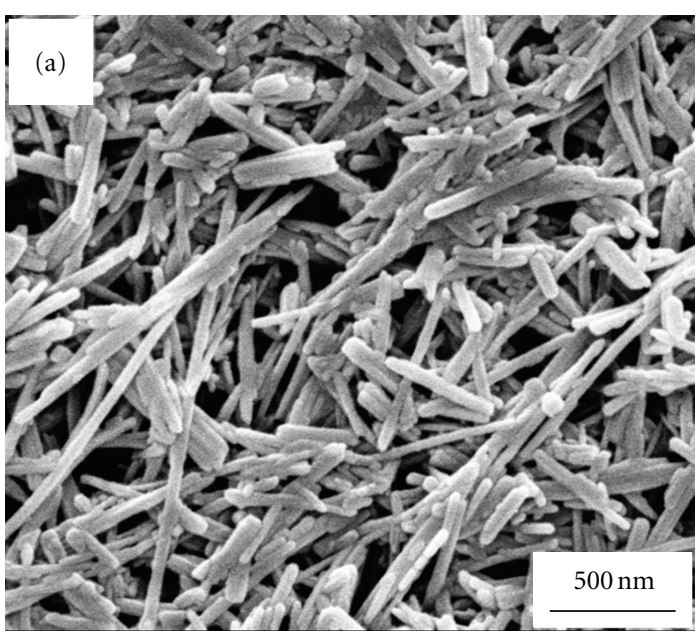

(a)

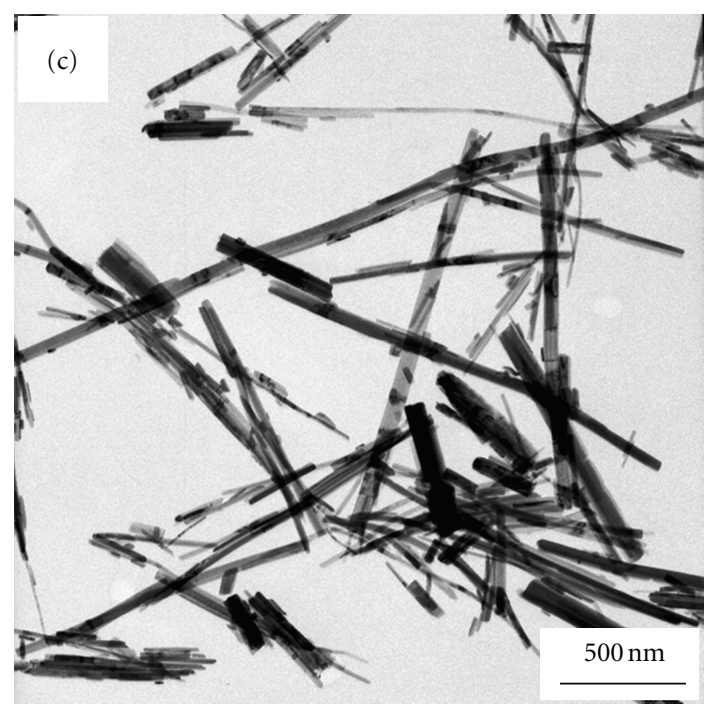

(c)

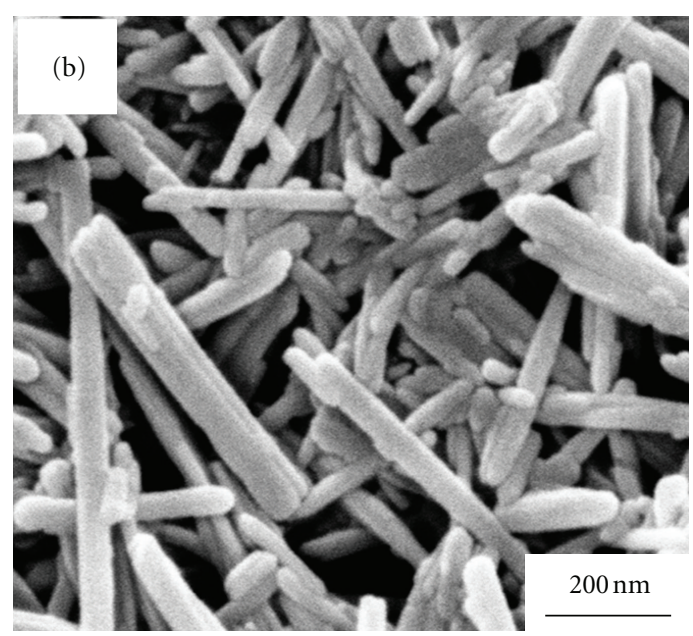

(b)

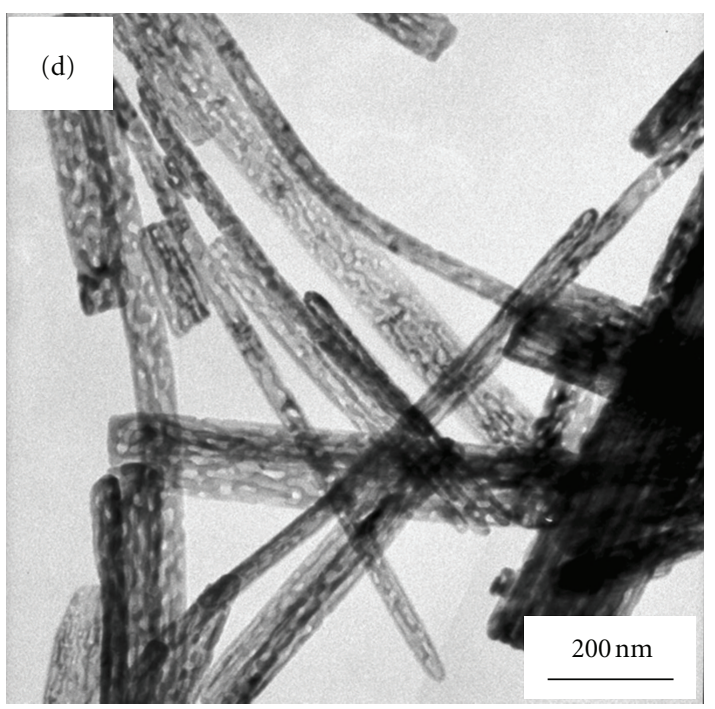

(d)

FIgURE 3: SEM (a, b) and TEM (c, d) images of the mesoporous $\mathrm{BiVO}_{4}$ nanorods with different magnifications.

\subsection{Photocatalytic Activity for Degradation of MB in Water}

3.2.1. Adsorption Performance of MB on the Photocatalysts. A preliminary test with regard to the adsorption performance of $\mathrm{MB}$ on the catalysts is performed to obtain a few sets of stand control data, and the test is performed in the dark and the corresponding results are listed in Table 2. Note that the degree of $\mathrm{MB}$ adsorption on the photocatalysts has relatively direct relationship with their SSA in the absence of irradiation except for P25. The larger the SSA is, the stronger the adsorption property is. As for P25, its SSA is the biggest among the three photocatalysts, while its adsorption rate is the smallest, which may be attributed to the rich hydroxyl groups on the surface of $\mathrm{TiO}_{2}$, and many $\mathrm{TiO}_{2}$ nanoparticles seriously aggregated together due to hydrogen bond and thus resulted in its adsorption rate decreasing toward $\mathrm{MB}[8,35,36]$. Besides the contribution from the effect of photocatalytic degradation, the total removal efficiency also comes from the assistance of the rapid attainment of adsorption equilibrium of the dye onto the photocatalysts. However, total removal of pollutant is enhanced dramatically by carrying out the photocatalytic degradation experiment under illumination of light. Especially, for the as-prepared mesoporous $\mathrm{BiVO}_{4}$ sample, its total removal for pollutant can be attributed to more than $71.2 \%$ of photodegradation capability in addition to the above-mentioned surface adsorption effect.

\subsubsection{Photocatalytic Activity for Degradation of $M B$ in Natural} Sunlight. The photocatalytic activities of the photocatalysts are evaluated by photocatalytic degradation of $\mathrm{MB}$ aqueous solution under the natural sunlight irradiation through monitoring the intensity of the characteristic absorption peak at $664 \mathrm{~nm}$ of MB. For comparison, the photocatalytic performance of P25, SSR-BiVO of MB without catalysis (sunlight only) are also investigated under the same experimental conditions. The removal 
TABLE 2: Preliminary test for the adsorption and degradation performances of $\mathrm{MB}$ on mesoporous $\mathrm{BiVO}_{4}$, nanorods, $\mathrm{SSR}^{-\mathrm{BiVO}} 4$ and $\mathrm{P} 25$, respectively.

\begin{tabular}{lccc}
\hline & $\mathrm{BiVO}_{4}$ nanorods & SSR-BiVO $_{4}$ & \multicolumn{2}{c}{$\mathrm{P} 25$} \\
\hline Initial concentration $\left(10^{-5} \mathrm{~mol} / \mathrm{L}\right)$ & 5 & 5 & 5 \\
Adsorption removal $^{\mathrm{a}}\left(10^{-5} \mathrm{~mol} / \mathrm{L} / \%\right)$ & $1.380 / 27.6$ & $0.570 / 11.4$ & $0.490 / 9.8$ \\
Photodegradation removal $^{\mathrm{b}}\left(10^{-5} \mathrm{~mol} / \mathrm{L} / \%\right)$ & $3.560 / 71.2$ & $0.585 / 11.7$ & $0.220 / 4.4$ \\
Total removal $^{c}\left(10^{-5} \mathrm{~mol} / \mathrm{L} / \%\right)$ & $4.940 / 98.8$ & $1.155 / 23.1$ & $0.710 / 14.2$ \\
\hline
\end{tabular}

${ }^{a}$ Total removal in the dark using photocatalyst.

${ }^{\mathrm{b}}$ Total removal - adsorption removal $=$ photodegradation removal.

${ }^{\mathrm{c}}$ Total removal under light illumination using photocatalyst.

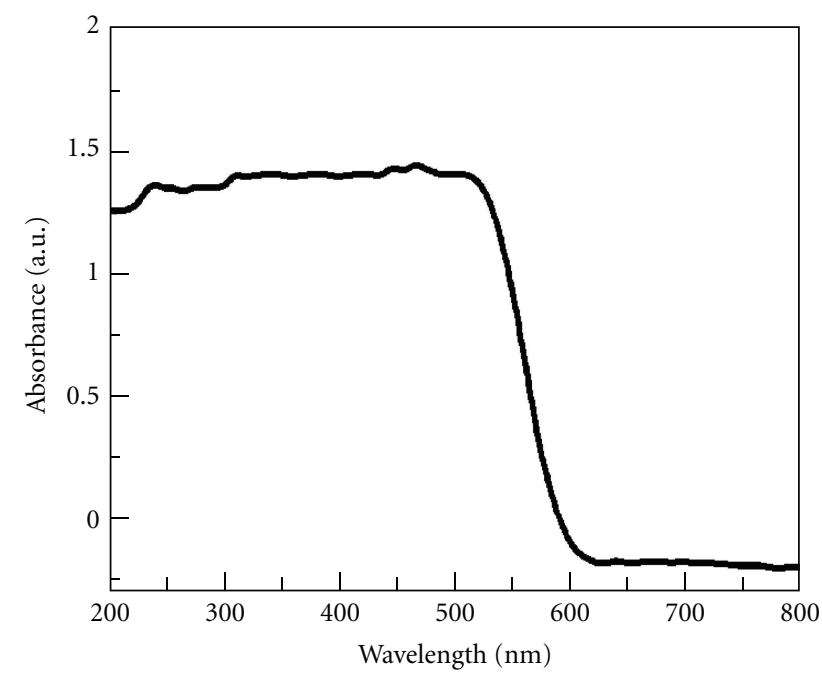

FIGURE 4: UV-Vis diffuse reflectance spectrum of the mesoporous $\mathrm{BiVO}_{4}$ nanorods.

efficiency of $\mathrm{MB}$ under different conditions is reported in Figure 5. It can be seen from Figure 5 that the photolysis of $\mathrm{MB}$ is extremely slow without a photocatalyst under sunlight illumination, which is in consistent with the reference [37]. The decoloration rate of $\mathrm{MB}$ solution in presence of P25 is higher than that of direct photolysis, while it is only $14.2 \%$ over a period of $180 \mathrm{~min}$, indicating that P25 is inactive under sunlight irradiation. The decrease of $\mathrm{MB}$ concentration in the presence of $\mathrm{SSR}^{-} \mathrm{BiVO}_{4}$ under sunlight is still small, only ca. $23.1 \%$ of MB was degraded in $180 \mathrm{~min}$, which is attributed to the poor adsorption performance and difficult migration of electron-hole pairs of SSR-BiVO ${ }_{4}$. However, the decoloration rate of MB increases significantly by substituting $\mathrm{SSR}-\mathrm{BiVO}_{4}$ for the mesoporous $\mathrm{BiVO}_{4}$ nanorods, and it reached $98.8 \%$ in $180 \mathrm{~min}$. The assynthesized $\mathrm{BiVO}_{4}$ nanorods are endowed with relatively higher SSA due to their 1D mesoporous rod-like structure, which provides richer active sites for $\mathrm{MB}$ adsorption and hence facilitates the enhancement in photocatalytic performance [38]. Nevertheless, the decolorizing of $\mathrm{MB}$ by the mesoporous $\mathrm{BiVO}_{4}$ nanorods is mainly attributed to the photocatalytic degradation rather than the simple adsorption.

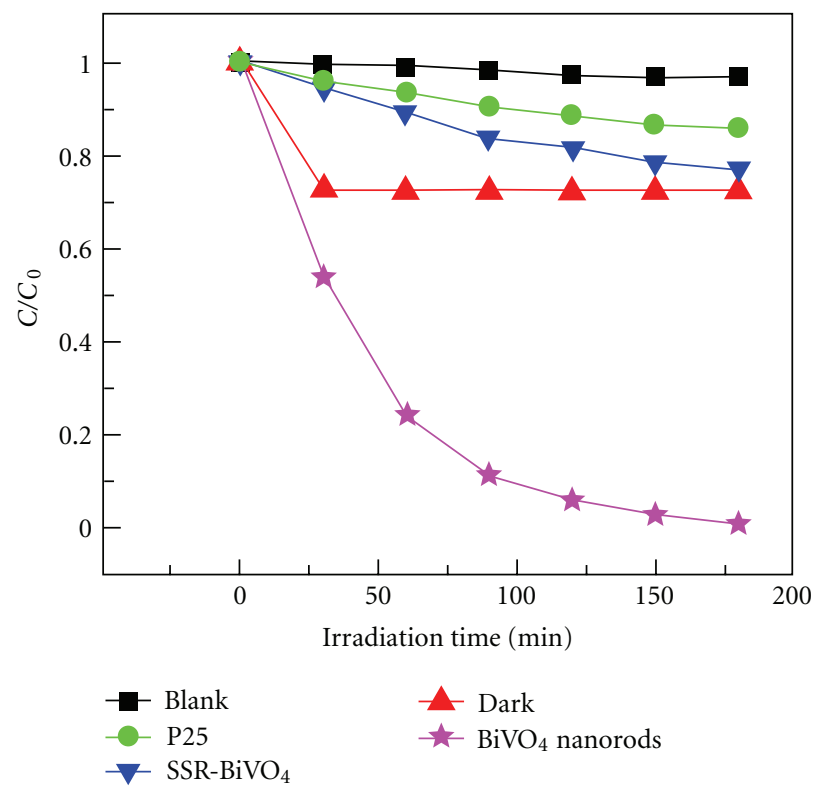

FIGURE 5: Photodegradation efficiencies of the MB as a function of irradiation time for different photocatalysts.

To obtain some information about photocatalytic degradation mechanism of $\mathrm{MB}$ by the mesoporous $\mathrm{BiVO}_{4}$ nanorods, a possible pathway of the photoelectrons transfer excited by sunlight was tentatively introduced. As is known to all, monoclinic $\mathrm{BiVO}_{4}$ has a suitable band structure to absorb visible light due to its hybrid $\mathrm{VB}$ formed by $\mathrm{Bi}_{6 \mathrm{~s}}$ and $\mathrm{O}_{2 \mathrm{p}}$ [18]. Based on the UV-Vis reflectance spectrum (Figure 4), the band gap of the mesoporous $\mathrm{BiVO}_{4}$ nanorods is calculated as $2.18 \mathrm{eV}$. Under the sunlight illumination, $\mathrm{BiVO}_{4}$ nanorods absorb the efficient photons $(\mathrm{h} v \geq 2.18 \mathrm{eV}$ ), and the electrons in the $\mathrm{VB}$ of $\mathrm{BiVO}_{4}$ are excited to $\mathrm{CB}$, and thus the $\mathrm{VB}$ of $\mathrm{BiVO}_{4}$ is rendered the corresponding holes (Figure 6). Simultaneously, the adsorbed dye is excited to singlet or triplet states (dye*) due to the absorbing properties of dye for light, even if the wavelength of the light is longer than $600 \mathrm{~nm}$ [16], and then the electrons coming from the dye are injected into the $\mathrm{CB}$ of $\mathrm{BiVO}_{4}$. Additionally, the mesoporous structure of $\mathrm{BiVO}_{4}$ nanorods is beneficial to the migration of the photogenerated carriers; thus the probability of electron-hole recombination was reduced. The electrons in the $\mathrm{VB}$ of $\mathrm{BiVO}_{4}$ nanorods and the holes in 


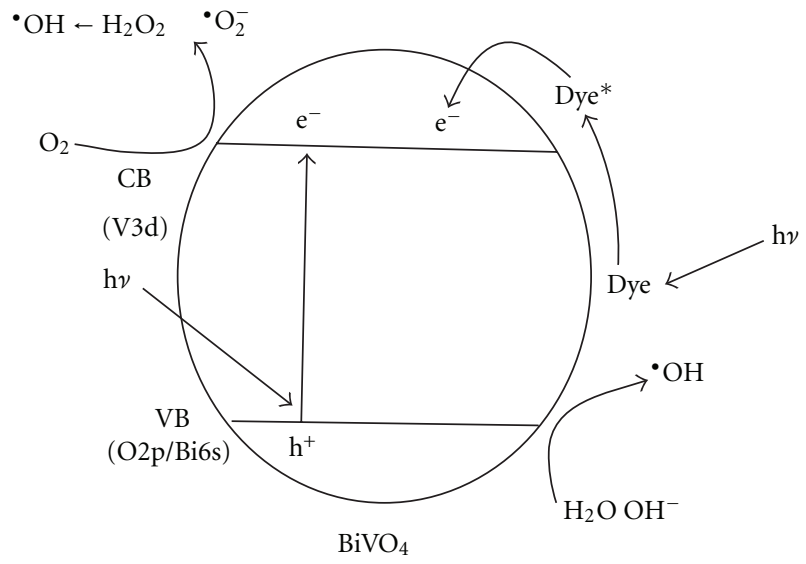

Figure 6: Photocatalytic mechanism for the mesoporous $\mathrm{BiVO}_{4}$ nanorods over MB.

the $\mathrm{CB}$ can participate in some reactions (as shown in (2)(8)) assisted by the $\mathrm{O}_{2}$ dissolved in water and $\mathrm{H}_{2} \mathrm{O}$, respectively, to generate many $\mathrm{OH}$ (Figure 6), which has strong oxidizing ability and is available to oxidize many organic pollutants $[39,40]$.

$$
\begin{gathered}
\mathrm{H}_{2} \mathrm{O} \longrightarrow \mathrm{H}^{+}+\mathrm{OH}^{-} \\
\mathrm{h}^{+}+\mathrm{OH}^{-} \longrightarrow \mathrm{OH}^{-} \\
\mathrm{e}^{-}+\mathrm{O}_{2} \longrightarrow \mathrm{O}_{2}{ }^{-} \\
\mathrm{O}_{2}{ }^{-}+\mathrm{H}^{+} \longrightarrow \mathrm{HO}_{2} \\
2 \mathrm{HO}_{2} \longrightarrow \mathrm{H}_{2} \mathrm{O}_{2}+\mathrm{O}_{2} \\
\mathrm{H}_{2} \mathrm{O}_{2}+\mathrm{O}_{2}{ }^{-} \longrightarrow{ }^{\cdot} \mathrm{OH}+\mathrm{OH}^{-}+\mathrm{O}_{2} \\
\mathrm{H}_{2} \mathrm{O}_{2}+\mathrm{h} v \longrightarrow 2 \cdot \mathrm{OH}
\end{gathered}
$$

To quantitatively understand the reaction kinetics of the $\mathrm{MB}$ degradation in our experiments, the LangmuirHinshelwood model expressed by (9) was applied. When the pollutant is in the millimolar concentration range, (9) is well established for photocatalysis experiments [41, 42]:

$$
-\ln \frac{C}{C_{0}}=k t
$$

where $C_{0}, C$, and $k$ indicate the concentrations of dye in solution at time 0 and $t$ and the apparent first-order rate constant, respectively. The reaction rate constant $(k)$ can be determined via the first-order linear fit. Efficient photocatalysts usually display high values of $k$, and the typical values of $k$ for the photocatalysts under sunlight irradiation are summarized in Table 3 . The results demonstrate that the photocatalytic activity of mesoporous $\mathrm{BiVO}_{4}$ nanorods is
TABLE 3: Effect of light sources upon the photocatalytic properties of different photocatalysts $\left(5 \times 10^{-5} \mathrm{~mol} / \mathrm{L}\right.$ dye concentration, 90 min light irradiation).

\begin{tabular}{lccc}
\hline Light sources & $\mathrm{BiVO}_{4}$ nanorods & $\mathrm{SSR}^{-B i V O}$ & $\mathrm{P} 25$ \\
\hline Sun light (\%) & 99.8 & 23.1 & 14.2 \\
Artificial light (\%) & 81.2 & 17.3 & 9.8 \\
\hline
\end{tabular}

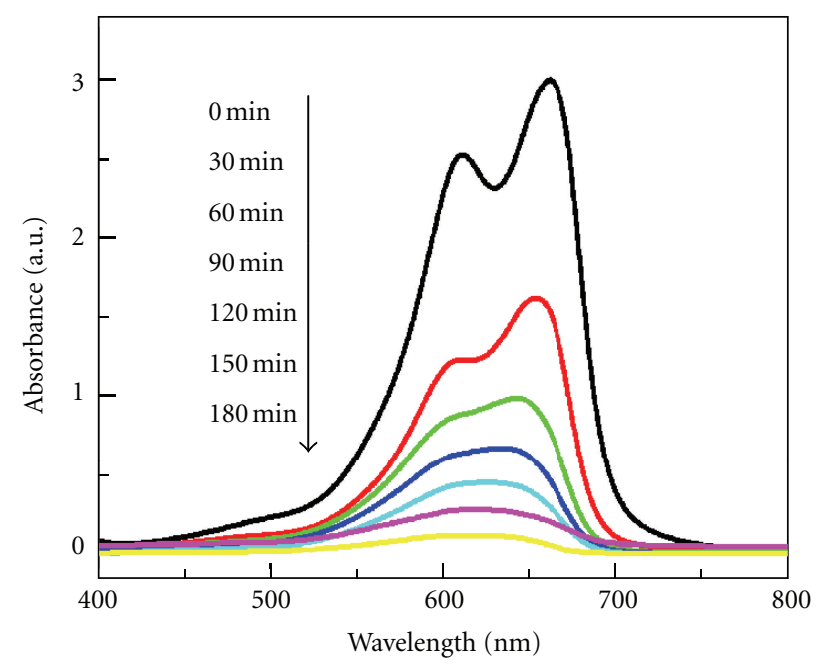

Figure 7: Temporal spectral changes of the $\mathrm{MB}$ in $\mathrm{BiVO}_{4}$ nanorods solution under sunlight irradiation; experimental conditions are otherwise identical to those of sunlight-irradiation $\mathrm{BiVO}_{4}$ dispersion in Figure 5.

much higher than those of SSR-BiVO 4 and P25, indicating its preferable photocatalytic performance.

In order to confirm that the MB solution was not simply decolorized during the degradation process, the absorption spectra of MB solutions as a function of irradiation time were further investigated (Figure 7). As seen in Figure 7, about 98.8\% of $\mathrm{MB}$ in $\mathrm{BiVO}_{4}$ nanorods slurry is degraded after being irradiated for $180 \mathrm{~min}$, and the spectral maximum shifted from 664 to $602 \mathrm{~nm}$. As is known to all, the peaks between 600 and $700 \mathrm{~nm}$ are assigned to the absorption of the conjugated $\pi$-system $[43,44]$, and the absorption band in this region is chosen to monitor the temporal concentration changes of $\mathrm{MB}$ in $\mathrm{BiVO}_{4}$ suspensions. Because of the existence of weak electron-donor substituents, methyl groups, $\mathrm{MB}$ is vulnerable to be attacked by electrophilic species $\left({ }^{\circ} \mathrm{OH}\right.$ or $\left.\mathrm{h}^{+}\right)$in the demethylation process, which is an important step in photocatalytic degradation process of MB [42]. When all or parts of the auxochromic groups (methyl or methylamine) degrade, the color of MB solutions becomes less intense, indicating that $\mathrm{MB}$ is $\mathrm{N}$-demethylated in a stepwise manner, which is confirmed by the gradual peak wavelength shifting toward the blue region, as shown in Figure 7.

3.2.3. Comparison of Photocatalytic Degradation of MB Solutions with Different Photocatalysts under Different Light Irradiation. The ultimate goal of the study by using the 
TABLE 4: Degradation rates of the $\mathrm{MB}$ over the mesoporous $\mathrm{BiVO}_{4}$ nanorods.

\begin{tabular}{lcccccc}
\hline & Raw solution & 1st use & 2nd use & 3rd use & 4th use & 5th use \\
\hline Absorbance (a.u.) & 2.981 & 0.006 & 0.012 & 0.009 & 0.009 & 0.012 \\
Degradation ratio (\%) & & 99.8 & 99.6 & 99.7 & 99.7 & 99.6 \\
\hline
\end{tabular}

mesoporous $\mathrm{BiVO}_{4}$ nanorods as photocatalyst waste water treatment is to apply this technology utilizing solar energy as the energy source. Accordingly, this work addresses the direct comparison of artificial and natural energy sources in order to apply this technique in the wastewater treatment plant. The comparison experiments were carried out by using other two different photocatalysts, SSR-BiVO 4 and P25.

Different light sources have an important effect on the photocatalytic properties of the photocatalysts. In order to further confirm that sun will be a promising and ideal light source, a $250 \mathrm{~W}$ medium pressure mercury arc lamp, whose main wavelength is $450 \mathrm{~nm}$, was also used in the experiment to replace natural sunlight to investigate the photocatalytic properties of all products in the laboratory in a similar quartz beaker. Table 3 shows the comparative results of the photocatalytic properties of different photcatalysts under different light sources irradiation $\left(5 \times 10^{-5} \mathrm{~mol} / \mathrm{L}\right.$ dye concentration, $90 \mathrm{~min}$ light irradiation). It could be seen from Table 1 that all photocatalysts showed higher photocatalytic properties under sunlight irradiation than artificial light, for which the reason could be attributed to the fact that the artificial light has the main wavelength of $450 \mathrm{~nm}$ while sunlight has partial UV light besides visible light. Thus it can be seen that sun will be a promising light source in the degradation process of dye. Moreover, it is both applicable and cost-saving to use sun as light source.

To confirm the stability of the high photocatalytic activity of the mesoporous $\mathrm{BiVO}_{4}$ nanorods, the photocatalytic degradation performance of $\mathrm{MB}$ over the circulated mesoporous $\mathrm{BiVO}_{4}$ nanorods under sunlight irradiation was investigated (Table 4). After five recycles for the photocatalytic degradation of $\mathrm{MB}$, the photocatalyst still exhibits high photocatalytic activity, which reveals that the as-prepared $\mathrm{BiVO}_{4}$ nanorods have high stability and do not photocorrode during the photocatalytic oxidation of the model pollutant MB. The high photocatalytic activity and stability of the mesoporous $\mathrm{BiVO}_{4}$ nanorods are especially beneficial to its practical application.

\section{Conclusions}

In conclusion, we first synthesized sunlight-driven mesoporous $\mathrm{BiVO}_{4}$ nanorods via a simple hydrothermal method. The as-prepared monoclinic $\mathrm{BiVO}_{4}$ photocatalyst exhibited high specific surface area of $10.67 \mathrm{~m}^{2} / \mathrm{g}$. The mesoporous $\mathrm{BiVO}_{4}$ nanorods presented strong absorptions in visible light region up to $660 \mathrm{~nm}$ as well as in the UV region. The band gap was estimated to be $c a .2 .18 \mathrm{eV}$. The excellent photodegradation rate of as-prepared $\mathrm{BiVO}_{4}$ nanorods was up to $98.8 \%$ in 180 min under natural sunlight irradiation, much higher than the $\mathrm{SSA}^{-\mathrm{BiVO}_{4}}(\mathrm{ca} .8 \%)$ and the commercial P25 (ca. 6\%) under the same conditions, owing to the higher specific surface area and the appropriate band gap. In addition, the hydrothermal route applied here is effective, convenient, low-cost, and environmentally friendly, and thus worth to be extended to other photocatalyst systems.

\section{Acknowledgments}

This work was financially supported by Natural Science Foundation of Anhui Province (no. 10040606Q07) and 2010 Young Teachers' Foundation of Anhui University of Technology (no. QZ201003).

\section{References}

[1] I. S. Cho, S. Lee, J. Hong-Noh et al., "Visible-light-induced photocatalytic activity in $\mathrm{FeNbO}_{4}$ nanoparticles," Journal of Physical Chemistry C, vol. 112, no. 47, pp. 18393-18398, 2008.

[2] A. Fujishima and K. Honda, "Electrochemical photolysis of water at a semiconductor electrode," Nature, vol. 238, no. 5358, pp. 37-38, 1972.

[3] J. Tang, Z. Zou, and J. Ye, "Efficient photocatalytic decomposition of organic contaminants over $\mathrm{CaBi}_{2} \mathrm{O}_{4}$ under visible-light irradiation," Angewandte Chemie-International Edition, vol. 43, no. 34, pp. 4463-4466, 2004.

[4] L. Xinping, H. Tao, H. Fuqiang, W. Wendeng, and S. Jianlin, "Photocatalytic activity of a Bi-based oxychloride $\mathrm{Bi}_{3} \mathrm{O}_{4} \mathrm{Cl}$," Journal of Physical Chemistry B, vol. 110, no. 48, pp. 2462924634, 2006.

[5] W. Yin, W. Wang, and S. Sun, "Photocatalytic degradation of phenol over cage-like $\mathrm{Bi}_{2} \mathrm{MoO}_{6}$ hollow spheres under visiblelight irradiation," Catalysis Communications, vol. 11, no. 7, pp. 647-650, 2010.

[6] S. Ouyang, Z. Li, Z. Ouyang, T. Yu, J. Ye, and Z. Zou, "Correlation of crystal structures, electronic structures, and photocatalytic properties in a series of Ag-based oxides: $\mathrm{AgAlO}_{2}$, $\mathrm{AgCrO}_{2}$, and $\mathrm{Ag}_{2} \mathrm{CrO}_{4}$," Journal of Physical Chemistry C, vol. 112, no. 8, pp. 3134-3141, 2008.

[7] J. Yu, Y. Zhang, and A. Kudo, "Synthesis and photocatalytic performances of $\mathrm{BiVO}_{4}$ by ammonia co-precipitation process," Journal of Solid State Chemistry, vol. 182, no. 2, pp. 223228, 2009.

[8] Y. Zhou, K. Vuille, A. Heel, B. Probst, R. Kontic, and G. R. Patzke, "An inorganic hydrothermal route to photocatalytically active bismuth vanadate," Applied Catalysis A, vol. 375, no. 1, pp. 140-148, 2010.

[9] H. Kato, M. Hori, R. Konta, Y. Shimodaira, and A. Kudo, "Construction of Z-scheme type heterogeneous photocatalysis systems for water splitting into $\mathrm{H}_{2}$ and $\mathrm{O}_{2}$ under visible light irradiation," Chemistry Letters, vol. 33, no. 10, Article ID CL040906, pp. 1348-1349, 2004.

[10] T. Guohui, F. Honggang, J. Liqiang, X. Baifu, and P. Kai, "Preparation and characterization of stable biphase $\mathrm{TiO}_{2}$ photocatalyst with high crystallinity, large surface area, and enhanced photoactivity," Journal of Physical Chemistry C, vol. 112, no. 8, pp. 3083-3089, 2008. 
[11] W. Liu, L. Cao, G. Su, H. Liu, X. Wang, and L. Zhang, "Ultrasound assisted synthesis of monoclinic structured spindle $\mathrm{BiVO}_{4}$ particles with hollow structure and its photocatalytic property," Ultrasonics Sonochemistry, vol. 17, no. 4, pp. 669674, 2010.

[12] L. R. Hou, C. Z. Yuan, and Y. Peng, "Synthesis and photocatalytic property of $\mathrm{SnO}_{2} / \mathrm{TiO}_{2}$ nanotubes composites," Journal of Hazardous Materials, vol. 139, no. 2, pp. 310-315, 2007.

[13] M.-L. Guan, D.-K. Ma, S.-W. Hu, Y.-J. Chen, and S.-M. Huang, "From hollow olive-shaped $\mathrm{BiVO}_{4}$ to n-p core-shell $\mathrm{BiVO}_{4} @ \mathrm{Bi}_{2} \mathrm{O}_{3}$ microspheres: controlled synthesis and enhanced visible-light-responsive photocatalytic properties," Inorganic Chemistry, vol. 50, no. 3, pp. 800-805, 2011.

[14] S. Tokunaga, H. Kato, and A. Kudo, "Selective preparation of monoclinic and tetragonal $\mathrm{BiVO}_{4}$ with scheelite structure and their photocatalytic properties," Chemistry of Materials, vol. 13, no. 12, pp. 4624-4628, 2001.

[15] M. Gotić, S. Musić, M. Ivanda, M. Šoufek, and S. Popović, "Synthesis and characterisation of bismuth(III) vanadate," Journal of Molecular Structure, vol. 744-747, special issue, pp. 535-540, 2005.

[16] L. Hou and C. Yuan, "Facile fabrication of taper-like $\mathrm{BiVO}_{4}$ nanorods with high photocatalytic property under sunlight irradiation," Advanced Materials Research, vol. 96, pp. 87-92, 2010.

[17] Y. Peng, L. R. Hou, and C. Z. Yuan, "Preparation and photocatalytic property of $\mathrm{BiVO}_{4}$ micro-rods with brick shape," Chinese Journal of Applied Chemistry, vol. 25, pp. 485488, 2008.

[18] A. Kudo, K. Omori, and H. Kato, "A novel aqueous process for preparation of crystal form-controlled and highly crystalline $\mathrm{BiVO}_{4}$ powder from layered vanadates at room temperature and its photocatalytic and photophysical properties," Journal of the American Chemical Society, vol. 121, no. 49, pp. 11459 11467, 1999.

[19] J. Su, L. Guo, S. Yoriya, and C. A. Grimes, "Aqueous growth of pyramidal-shaped $\mathrm{BiVO}_{4}$ nanowire arrays and structural characterization: application to photoelectrochemical water splitting," Crystal Growth and Design, vol. 10, no. 2, pp. 856861, 2010.

[20] R. Strobel, H. J. Mez, S. E. Pratsinis et al., "Brilliant yellow, transparent pure, and $\mathrm{SiO}_{2}$-coated $\mathrm{BiVO}_{4}$ nanoparticles made in flames," Chemistry of Materials, vol. 20, no. 20, pp. 63466351, 2008.

[21] D. Ke, T. Peng, L. Ma, P. Cai, and K. Dai, "Effects of hydrothermal temperature on the microstructures of $\mathrm{BiVO}_{4}$ and its photocatalytic $\mathrm{O}_{2}$ evolution activity under visible light," Inorganic Chemistry, vol. 48, no. 11, pp. 4685-4691, 2009.

[22] Y. Sun, Y. Xie, C. Wu, and R. Long, "First experimental identification of $\mathrm{BiVO}_{4} \cdot 0.4 \mathrm{H}_{2} \mathrm{O}$ and its evolution mechanism to final monoclinic $\mathrm{BiVO}_{4}$," Crystal Growth and Design, vol. 10, no. 2, pp. 602-607, 2010.

[23] M. Bielska and J. Szymanowski, "Removal of methylene blue from waste water using micellar enhanced ultrafiltration," Water Research, vol. 40, no. 5, pp. 1027-1033, 2006.

[24] M. Muruganandham and M. Swaminathan, "Solar photocatalytic degradation of a reactive azo dye in $\mathrm{TiO}_{2}$-suspension," Solar Energy Materials and Solar Cells, vol. 81, no. 4, pp. 439457, 2004.

[25] J. Yu, Y. Zhang, and A. Kudo, "Synthesis and photocatalytic performances of $\mathrm{BiVO}_{4}$ by ammonia co-precipitation process," Journal of Solid State Chemistry, vol. 182, no. 2, pp. 223228, 2009.
[26] A. W. Sleight, H. Y. Chen, A. Ferretti, and D. E. Cox, "Crystal growth and structure of $\mathrm{BiVO}_{4}$," Materials Research Bulletin, vol. 14, no. 12, pp. 1571-1581, 1979.

[27] R. M. Mohamed, A. A. Ismail, I. Othman, and I. A. Ibrahim, "Preparation of $\mathrm{TiO}_{2}-\mathrm{ZSM}-5$ zeolite for photodegradation of EDTA," Journal of Molecular Catalysis A, vol. 238, no. 1-2, pp. 151-157, 2005.

[28] Y. Shen, M. Huang, Y. Huang, J. Lin, and J. Wu, "The synthesis of bismuth vanadate powders and their photocatalytic properties under visible light irradiation," Journal of Alloys and Compounds, vol. 496, no. 1-2, pp. 287-292, 2010.

[29] M. Shang, W. Wang, L. Zhou, S. Sun, and W. Yin, "Nanosized $\mathrm{BiVO}_{4}$ with high visible-light-induced photocatalytic activity: ultrasonic-assisted synthesis and protective effect of surfactant," Journal of Hazardous Materials, vol. 172, no. 1, pp. 338344, 2009.

[30] X. Zhang, Z. Ai, F. Jia, L. Zhang, X. Fan, and Z. Zou, "Selective synthesis and visible-light photocatalytic activities of $\mathrm{BiVO}_{4}$ with different crystalline phases," Materials Chemistry and Physics, vol. 103, no. 1, pp. 162-167, 2007.

[31] F. Dong, Q. Wu, J. Ma, and Y. Chen, "Mild oxide-hydrothermal synthesis of different aspect ratios of monoclinic $\mathrm{BiVO}_{4}$ nanorods tuned by temperature," Physica Status Solidi A, vol. 206, no. 1, pp. 59-63, 2009.

[32] J. Tang, Z. Zou, and J. Ye, "Efficient photocatalytic decomposition of organic contaminants over $\mathrm{CaBi}_{2} \mathrm{O}_{4}$ under visible-light irradiation," Angewandte Chemie-International Edition, vol. 43, no. 34, pp. 4463-4466, 2004.

[33] J. Yu, W. Liu, and H. Yu, "A one-pot approach to hierarchically nanoporous titania hollow microspheres with high photocatalytic activity," Crystal Growth and Design, vol. 8, no. 3, pp. 930-934, 2008.

[34] L. R. Hou, C. Z. Yuan, and Y. Peng, "Preparation and photocatalytic property of sunlight-driven photocatalyst $\mathrm{Bi}_{38} \mathrm{ZnO}_{58}$," Journal of Molecular Catalysis A, vol. 252, no. 1-2, pp. 132-135, 2006.

[35] J. Kirchnerova, M. L. Herrera Cohen, C. Guy, and D. Klvana, "Photocatalytic oxidation of n-butanol under fluorescent visible light lamp over commercial $\mathrm{TiO}_{2}$ (Hombicat UV100 and Degussa P25)," Applied Catalysis A, vol. 282, no. 1-2, pp. 321332, 2005.

[36] K. A. Michalow, D. Logvinovich, A. Weidenkaff et al., "Synthesis, characterization and electronic structure of nitrogendoped $\mathrm{TiO}_{2}$ nanopowder," Catalysis Today, vol. 144, no. 1-2, pp. 7-12, 2009.

[37] Y. Zhou, K. Vuille, A. Heel, B. Probst, R. Kontic, and G. R. Patzke, "An inorganic hydrothermal route to photocatalytically active bismuth vanadate," Applied Catalysis A, vol. 375, no. 1, pp. 140-148, 2010.

[38] X. Meng, L. Zhang, H. Dai, Z. Zhao, R. Zhang, and Y. Liu, "Surfactant-assisted hydrothermal fabrication and visiblelight-driven photocatalytic degradation of methylene blue over multiple morphological $\mathrm{BiVO}_{4}$ single-crystallites," Materials Chemistry and Physics, vol. 125, no. 1-2, pp. 59-65, 2011.

[39] Z. Zhang, W. Wang, M. Shang, and W. Yin, "Photocatalytic degradation of rhodamine $\mathrm{B}$ and phenol by solution combustion synthesized $\mathrm{BiVO}_{4}$ photocatalyst," Catalysis Communications, vol. 11, no. 11, pp. 982-986, 2010.

[40] Z. Z. Qin, Z. L. Liu, Y. B. Liu, and K. D. Yang, "Synthesis of $\mathrm{BiYO}_{3}$ for degradation of organic compounds under visiblelight irradiation," Catalysis Communications, vol. 10, no. 12, pp. 1604-1608, 2009.

[41] S. Sun, W. Wang, L. Zhang, L. Zhou, W. Yin, and M. Shang, "Visible light-induced efficient contaminant removal 
by $\mathrm{Bi}_{5} \mathrm{O}_{7} \mathrm{I}$," Environmental Science and Technology, vol. 43, no. 6, pp. 2005-2010, 2009.

[42] M. Shang, W. Wang, S. Sun, J. Ren, L. Zhou, and L. Zhang, "Efficient visible light-induced photocatalytic degradation of contaminant by spindle-like PANI/BiVO ${ }_{4}$," Journal of Physical Chemistry C, vol. 113, no. 47, pp. 20228-20233, 2009.

[43] S. Kohtani, M. Koshiko, A. Kudo et al., "Photodegradation of 4-alkylphenols using $\mathrm{BiVO}_{4}$ photocatalyst under irradiation with visible light from a solar simulator," Applied Catalysis B, vol. 46, no. 3, pp. 573-586, 2003.

[44] S. Kohtani, J. Hiro, N. Yamamoto, A. Kudo, K. Tokumura, and R. Nakagaki, "Adsorptive and photocatalytic properties of Agloaded $\mathrm{BiVO}_{4}$ on the degradation of 4-n-alkylphenols under visible light irradiation," Catalysis Communications, vol. 6, no. 3, pp. 185-189, 2005. 


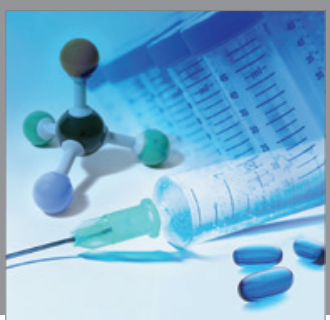

International Journal of

Medicinal Chemistry

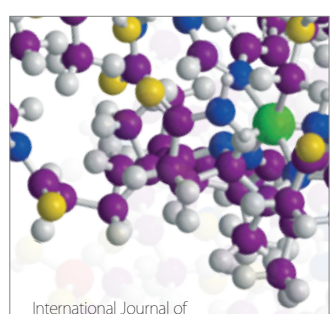

Carbohydrate Chemistry

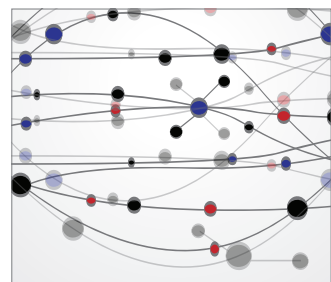

The Scientific World Journal
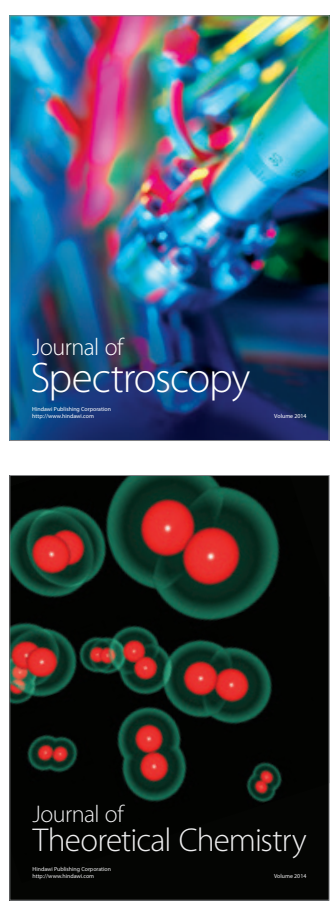
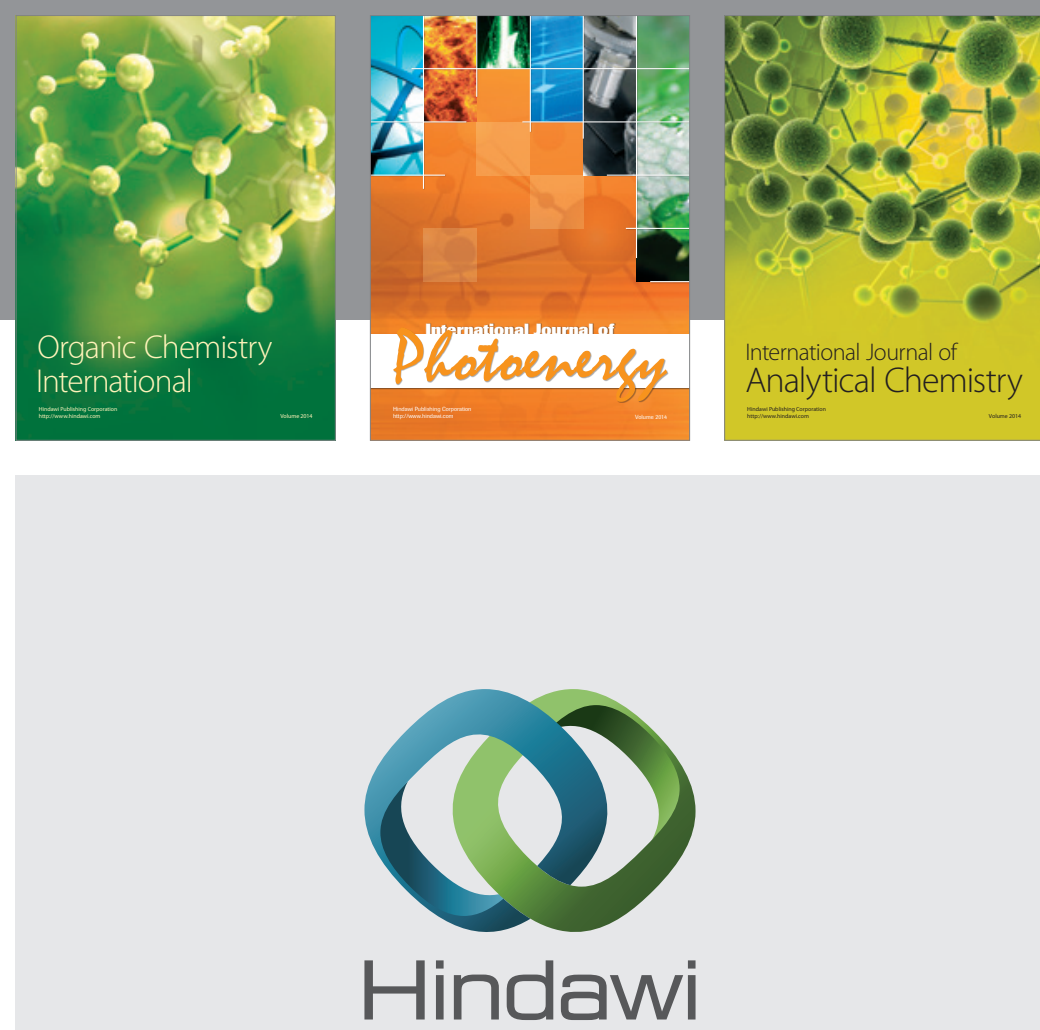

Submit your manuscripts at

http://www.hindawi.com
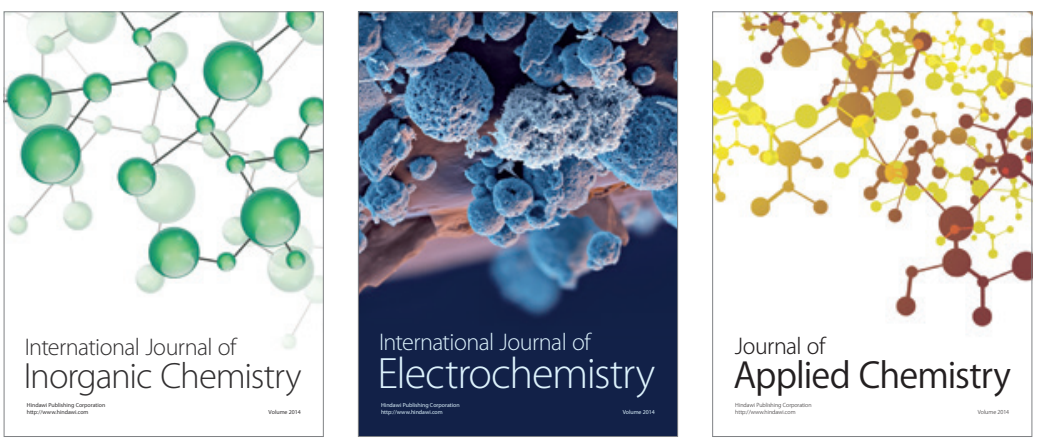

Journal of

Applied Chemistry
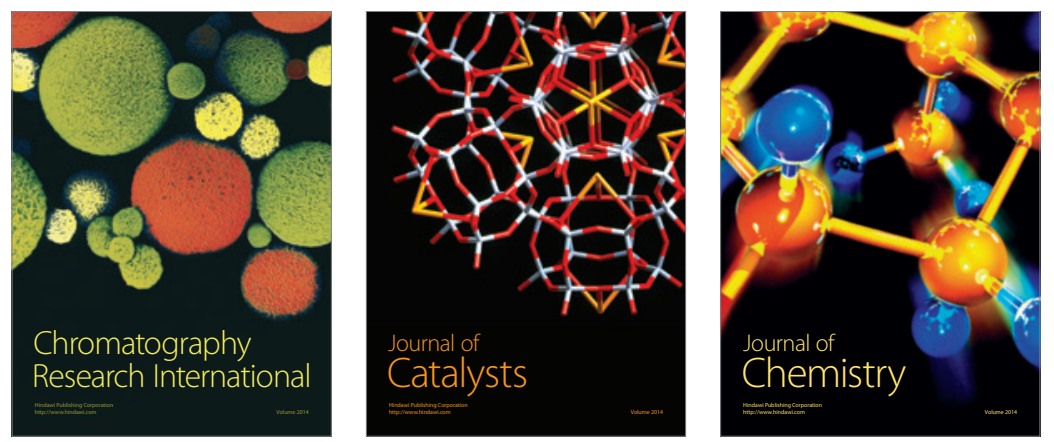
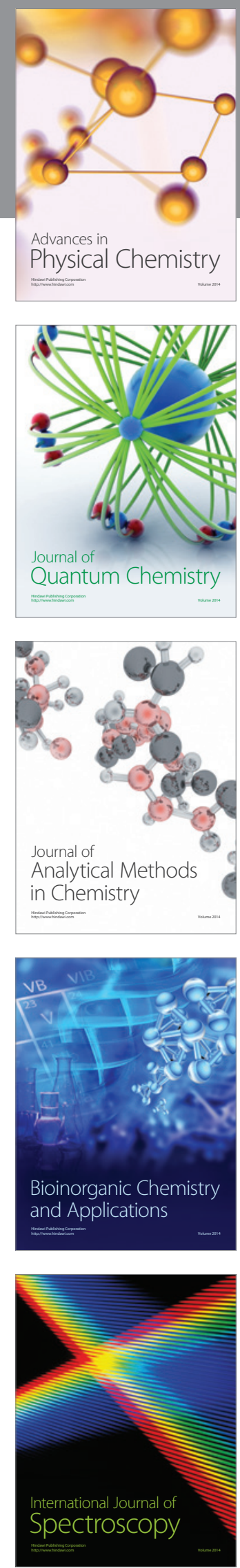